\title{
Efectos no Intencionados de Fungicidas para Controlar el Tizón Tardío de la Papa en la Proliferación del Áfido Verde del Duraznero, Myzus persicae (Sulzer)
}

\author{
Jorge Mario Ruano Rossil ${ }^{1}$
}

Resumen. Los pesticidas han brindado muchos beneficios a la humanidad, aunque su utilización también ha provocado efectos negativos en el ambiente por contaminación directa y por la alteración ecológica de muchas especies de organismos. En el aspecto agrícola, un inconveniente de la aplicación intensiva de pesticidas ha sido la eliminación de enemigos naturales de las plagas agrícolas más importantes. Un ejemplo de este aspecto es el creciente aumento del áfido verde del duraznero (AVD), Myzus persicae (Sulzer), en los campos de Minnesota y Dakota del Norte de los Estados Unidos en las últimas dos décadas, que ha correlacionado con un aumento en el número de enfermedades virales de la papa, Solanum tuberosum (Linnaeus). Esto se ha debido a la aplicación intensiva de fungicidas foliares en la papa para controlar el tizón temprano, Alternaria solani (Sorauer), y el tizón tardío, Phytophthora infestans (Mont.) DeBary. Dicha aplicación intensiva ha alterado el balance natural de un grupo de hongos entomopatógenos perteneciente al orden Entomophthorales. A finales de la década de los 90 `s, esta situación promovió una investigación sobre la aplicación de fungicidas de diferente composición química (por ejemplo etilén-bis-ditiocarbamatos, EBDCs o ditiocarbamatos y a base de clorotalonil), de diferente regímenes de aplicación y en diferentes sistemas de producción agrícola (tierras irrigadas o no irrigadas). Al final de este estudio, todos los fungicidas evaluados, sin importar su composición química o régimen de aplicación, fueron nocivos para los Entomophthorales. Sin fungicidas foliares, las poblaciones del AVD alcanzaron $30 \%$ de micosis (enfermedad o nivel de infección) al final de las primeras 4 semanas después de la llegada del áfido a la planta. Sin embargo, cuando se aplicaron fungicidas foliares para controlar el tizón tardío, tres cosas se observaron: una reducción en la tasa de infección de los AVD, una baja en su tasa de mortalidad, así como un aumento en el número total de áfidos a lo largo de la estación de cultivo. Aunque todos los fungicidas foliares exhibieron un efecto fungicida poderoso, los fungicidas a base de clorotalonil tendieron a ser más efectivos en reducir la micosis de AVD causadas por Entomophthorales que los fungicidas a base de EBDC. Una supresión en los niveles de micosis implicó tasas de mortalidad mucho más bajas en la población del áfido, lo que condujo a alcanzar densidades de áfidos ápteros (sin alas) mucho más elevadas. Las densidades más elevadas de áfidos ápteros condujeron a mayores producciones de áfidos alados (con alas). Estas dos últimas variables fueron asociadas a patrones de dispersión diferenciales donde la efectividad del fungicida aplicado varió con respecto a las condiciones climáticas prevalentes, así como a las especies de hongos entomopatógenos predominantes en cada estación del cultivo. Si bien el efecto de los fungicidas sobre la dinámica de crecimiento del AVD no ocurre intencionalmente, pues los fungicidas no fueron diseñados para afectar al insecto en sí, este efecto indeseable ocurre, pero a través de la supresión de uno de los grupos más importantes de hongos entomopatógenos que parasitan al áfido.

Palabras clave: Clorotalonil, control biológico; EBDCs; Entomophthorales; hongos entomopatógenos; Myzus persicae; Phytophthora infestans.

\section{Unintended Effects of Potato Late Blight Fungicides over the Proliferation of Green Peach Aphid, Myzus persicae (Sulzer)}

Abstract. Pesticides have brought multiple benefits to humanity, but their use has also caused negative effects to the environment through direct contamination and ecological disruption of many species of organisms. Intensive pesticide use has eliminated natural enemies of the most important agricultural pests. An example of this phenomenon can be found in the United States, specifically in Minnesota and North Dakota's agricultural fields with an ever-increasing number of green peach aphid (GPA), Myzus persicae (Sulzer) over the last two

\footnotetext{
${ }^{1}$ Departamento de Investigaciones, División de Ciencia y Tecnología, Centro Universitario de Occidente, Universidad de San Carlos de Guatemala, Calle Rodolfo Robles, 29-99 Zona 1, Quetzaltenango, Quetzaltenango, Guatemala. Correo electrónico: georcci@gmail.com
} 
decades, which correlates with an increased number of viral diseases on potato, Solanum tuberosum (Linnaeus). This is due to the intensive application of foliar fungicides on potato to control early and late blight diseases, Alternaria solani (Sorauer) and Phytophthora infestans (Mont.) DeBary, respectively. Such intensive application has altered the natural balance of a group of entomopathogenic fungi of the order Entomophthorales. At the end of the 1990s, this situation promoted the study on fungicide applications of different chemical composition (i.e., ethylene-bis-dithiocarbamates, EBDCs or dithiocarbamates and chlorothalonil-based fungicides), in different application regimens, and in different agricultural production systems (irrigated or non-irrigated). At the conclusion of this study, all fungicides evaluated, no matter what their chemical composition or application regimen, were detrimental to Entomophthorales. Without foliar fungicides, GPA populations reached $30 \%$ mycosis (disease or infection level) by the end of the first 4 weeks after the aphid had arrived to the plant. However, when foliar fungicides were applied to control Late Blight, three things were observed: a reduction of GPA infection rate, a lower aphid mortality rate, and an increase of aphid numbers throughout the growing season. Although all foliar fungicides exhibited a strong fungicidal effect, chlorothalonil-based fungicides were more effective than EBDC-based fungicides in suppressing GPA's mycosis caused by Entomophthorales. Suppressed mycoses elicited lower aphid population mortality rates, which led to higher apterus (wingless) aphid densities. Higher densities of apterus aphids led to higher productions of winged (alate) aphids. These latter two variables were associated with differential dispersal patterns, where the effectiveness of the applied fungicide varied according to prevalent climatic conditions as it did with the predominant species of entomopathogenic fungi of each growing season. It is clear that the fungicidal effect on the population dynamics of GPA, although unintended since it has not been designed to affect the insect per se, does occur, but via suppression of some of the aphid's most important parasites, a select group of entomopathogenic fungi.

Key words: Biological control, Chlorothalonil, EBDCs; entomopathogenic fungi; Entomophthorales; Myzus persicae, Phytophthora infestans.

\section{Introducción}

\section{Plaguicidas y su aporte al desarrollo de insectos plaga}

Los avances agrícolas experimentados en los años 1940s, entre los que se encuentran la aparición de los primeros plaguicidas modernos, sentaron como precedente el lanzamiento de la revolución verde de los años 1960s, la cual perseguía aumentar la producción agrícola. Aunque no se puede ignorar que los plaguicidas han brindado muchos beneficios a la humanidad, entre ellos la disminución en la incidencia de ciertas enfermedades, no se puede negar que su aparición también ha estado colmada de efectos negativos.

Los primeros efectos nocivos documentados de plaguicidas modernos fueron popularizados por insecticidas que contaminaban el suelo, agua $y$ causaban mortalidad en peces, aves y humanos. Un efecto de los insecticidas también fue vinculado con la proliferación inusual de insectos que se convertían en importantes plagas. EI DDT es un ejemplo clásico de esta situación, su uso excesivo en la agricultura aceleró en ciertos insectos la resistencia a su aplicación, permitiendo que se convirtieran en verdaderas plagas agrícolas. Un ejemplo de la resistencia al DDT fue el escarabajo colorado de la papa, Leptinotarsa decemlineata (Say) en 1952. Posteriormente, ese mismo insecto desarrolló resistencia a los organoclorados, i.e., el insecticida dieldrin, así como los compuestos organofosforados y carbamatos (Quinton 1955, Hofmaster et al. 1967, Forgash 1985). Otro aspecto importante fue que el uso intensivo de insecticidas eliminaba a los enemigos naturales importantes de ciertos insectos, contribuyendo a que sus poblaciones incrementaran rápidamente. Un ejemplo de este aspecto es el carbaryl (carbamato) y el malathion (organofosforado). Ambos resultaron muy tóxicos para los depredadores y parasitoides del áfido verde del duraznero (AVD) Myzus persicae (Sulzer) (Horn 1983). Los insecticidas también han sido tóxicos a hongos entomopatógenos benéficos que son agentes biológicos de plagas agrícolas, principalmente de insectos y arácnidos.

Los herbicidas y fungicidas también inhiben el crecimiento de este grupo de enemigos naturales en la superficie de las plantas así como en el suelo (Pell et al. 2010). De estos tres tipos de plaguicidas, los fungicidas son los más dañinos a hongos patógenos benéficos (Klingen y Haukeland 2006). 
Ruano Rossil. Efectos no Intencionados de Fungicidas para Controlar el Tizón Tardío de la Papa

\section{El áfido verde del duraznero y el cultivo de la papa}

En los últimos 20 años, en los campos agrícolas de Minnesota y Dakota del Norte en Los Estados Unidos se ha observado un aumento de enfermedades virales en el cultivo de la papa, Solanum tuberosum (L.), correlacionadas con el aumento inusual de las poblaciones del AVD, también conocido como el pulgón verde del duraznero (durazno) o melocotonero (melocotón). Este insecto cosmopolita y de naturaleza migrante, además de causar daños a la planta de papa (su hospedero secundario), es el vector más importante del virus de mosaico $Y$ (PVY) y del enrollamiento de la hoja (PLRV). Normalmente, este insecto se alimenta de las partes inferiores de la planta de papa (Raman 1985).

El aumento del AVD había sido vinculado al uso intensivo de insecticidas, principalmente los aplicados para controlar otras plaga como el escarabajo colorado de la papa y el saltahojas verde o chicharrita de la papa, Empoasca fabae (Harris). El aumento se debia a que estos insecticidas favorecían la eliminación de sus enemigos naturales como las avispas parasitoides y depredadores como los escarabajos coccinélidos, así como la aceleración de la selección y el desarrollo de resistencia a sus mismas aplicaciones.

EI AVD es hoy resistente a la mayoría de los insecticidas. Con el control diezmado de parasitoides y depredadores y con una resistencia marcada a los insecticidas, el AVD puede duplicar su población cada 2 a 3 días (Radcliffe et al. 1996). Sin embargo, las aplicaciones de fungicidas agrícolas en papa para controlar enfermedades de la hoja como el tizón temprano y el tizón tardío también contribuyen a alterar el balance natural de ciertos hongos entomopatógenos pertenecientes al orden Entomophthorales, que se consideran benéficos porque pueden controlar al AVD (Lagnaoui y Radcliffe 1998).

\section{Los entomophthorales y fungicidas utilizados para controlar el tizón tardío de la papa}

Desde la apareción de la resistencia de aislamientos de Phytophthora infestans (Mont.) DeBary al fungicida metalaxyl (Ridomil ${ }^{\circledR}$ ) en la década de los 1990's, el uso constante de fungicidas protectivos para controlarlo creció vertiginosamente (Goodwin et al. 1996). P. infestans es el organismo patógeno causante del tizón tardío de la papa.

Los Entomophthorales son un orden de hongos entomopatógenos que parasitan y eventualmente matan a los áfidos, entre ellos al AVD. Los Entomophthorales provocan epizootias pronunciadas en muchos insectos (Milner et al. 1980, Dedryver 1980, Soper y MacLeod 1981, Fedorenko 1985, Feng et al. 1991, Wraight et al. 1993, Feng et al. 1998, Nielsen y Hajek 2005).

Epizootia es un término equivalente a epidemia que define una enfermedad contagiosa que ataca a un número inusual de organismos al mismo tiempo y lugar y que se propaga con rapidez (Fuxa y Tanada 1987, Tanada y Kaya 1993). Los Entomophthorales es uno de los grupos más importantes de agentes biológicos para el control del AVD. Por su condición de hongos, estos no están exentos de sufrir el efecto tóxico de los fungicidas comerciales utilizados para controlar los tizones en la papa. Los Entomophthorales se encuentran comúnmente presentes en el ambiente en forma de esporas resistentes, principalmente en suelos, así como en remanentes de cadáveres de insectos y en vegetación en descomposición (American Public Health Association 2004).

En el medio oeste de los Estados Unidos y durante la estación del cultivo de la papa, la supresión de Entomophthorales por los fungicidas foliares, contribuye a que el número de AVD incremente notablemente. A finales de los 1990s, surgió en Minnesota el interés por descubrir hasta donde estos químicos pudieran estar limitando la acción biológica de este grupo de hongos sobre el AVD. promovió un estudio exhaustivo de fungicidas de diferente composición química (por ejemplo etilén-bisditiocarbamatos, EBDCs o ditiocarbamatos y de base clorotalonil), de diferente régimen de aplicación y en diferentes sistemas de producción agrícola (tierras irrigadas o no irrigadas).

Para la finalidad de este artículo, se discutirán los puntos más relevantes de la investigación de RuanoRossil en 2001, utilizando únicamente como ejemplos los fungicidas comunes para las estaciones de cultivo de 1998 y 1999, denominándoseles tan solo como ditiocarbamatos y a base de clorotalonil. 


\section{Descripción y Discusión}

\section{Efecto de fungicidas foliares sobre la acumulación del inóculo en el campo}

Entre los puntos más relevantes de la investigación de Ruano-Rossil en 2001 se encontró que todos los fungicidas evaluados, sin importar su composición química o régimen de aplicación, fueron nocivos para los hongos entomopatógenos del orden Entomophthorales. Se determinó que las infecciones fungales en aquellos áfidos que no tuvieron contacto con fungicidas, iniciaban con los primeros insectos detectados en el follaje de la papa y cuando sus poblaciones eran aún muy bajas. Es por ello que las primeras cuatro semanas después de su llegada o primer avistamiento del áfido sobre el follaje de la papa se consideran cruciales para la acumulación del inóculo (= insectos afectados por infecciones fungales que las diseminan a insectos sanos).

Sin la interferencia de fungicidas, la micosis en la población del AVD puede alcanzar niveles por encima del 30\% (nivel de enfermedad o infección) ya para finales de las primeras 4 semanas después de su llegada a la planta. Este porcentaje de micosis se consideró suficientemente alto como para producir altas mortalidades en la población del áfido y capaz de devastar su número a niveles casi imperceptibles. Sin embargo, cuando se aplicaron fungicidas foliares para controlar el tizón tardío de la papa, la población de AVD se mantuvo casi libre de infecciones causadas por Entomophthorales. Fue en estas primeras cuatro semanas donde el nivel de infección en áfidos provenientes de tratamientos con ditiocarbamatos y clorotalonil no pudo exceder $2.5 \%$ para las estaciones de cultivo de 1998 y 1999.

\section{A bajos niveles de infección en el áfido, menor mortalidad del insecto}

Desde la aparición del AVD, y hasta unas 5 semanas después de su llegada al campo, los Ditiocarbamatos y fungicidas a base de clorotalonil fueron muy eficientes en retrasar las micosis provocadas por hongos Entomophthorales comparados con aquellos áfidos que se encontraban en parcelas donde no se aplicó ningún fungicida. El efecto del fungicida sobre estos hongos entomopatógenos fue tan intenso que el porcentaje de micosis se mantuvo por debajo del $5.0 \%$, lo que provocó que el AVD se mantuviera casi libre de enfermedades durante este periodo y no fue sino hasta la sexta semana cuando los niveles de infección pudieron elevarse a $30 \%$. Esto se tradujo en que para la séptima semana después de la llegada del áfido al campo, las poblaciones del AVD en los tratamientos con ditiocarbamatos, clorotalonil y el testigo sin fungicida se encontraran en 143.4, 79.5 y 8.1 áfidos por hoja para 1998, y 30.6, 34.3 y 4.3 áfidos por hoja para 1999, respectivamente.

Al analizar el área total bajo la curva de progreso de la enfermedad o parasitismo en estos áfidos (= AUDPC = Area Under Disease Progress Curve, por sus siglas en inglés) para las estaciones de cultivo 1998 y 1999 y para los fungicidas en común (ditiocarbamatos y a base de clorotalonil) en comparación con sus testigos sin fungicida, se cuantificó que al aplicar fungicidas la micosis de los áfidos se redujo $80 \%$ o en otras palabras la infección en los áfidos provenientes de los tratamientos ditiocarbamatos y clorotalonil solo fue de 0.2 veces la infección promedio alcanzada en los áfidos provenientes de los testigos sin fungicida para ambas estaciones de cultivo (Figuras 1A, 1B) (Ruano-Rossil 2001).

\section{A menor mortalidad del insecto, densidades más elevadas del áfido}

Las micosis en los áfidos de las parcelas sin fungicida pueden iniciarse desde el avistamiento de los primeros insectos en el campo a densidades tan bajas como 0.2 AVD por hoja. Sin embargo, con la aplicación de fungicidas se reduce la tasa de infección (micosis) en el AVD. Como consecuencia de esto, se detectaron dos respuestas en la población de áfidos:

1) Una disminución en la tasa de mortalidad del áfido como efecto de la supresión y retraso de las infecciones causadas por Entomophthorales que se extendió 5 semanas después de su primera detección en el campo en 1998 y 1999. Al promediar ambas estaciones de cultivo en estas primeras 5 semanas se determinó que el $98.8 \%$ de los áfidos colectados donde se aplicó fungicidas no mostraron infecciones por Entomophthorales, comparados con el $52.5 \%$ de los áfidos colectados de testigos sin fungicida. Ya para esta semana, los Entomophthorales habían infringido mortalidades de 67.5 y $27.5 \%$ en los testigos sin fungicida en 1998 y 1999, 
Ruano Rossil. Efectos no Intencionados de Fungicidas para Controlar el Tizón Tardío de la Papa

respectivamente, impactando el número total de áfidos por día en ambas estaciones de cultivo (Figuras 1C, 1D) (Ruano-Rossil 2001).

No fue sino hasta la séptima semana después del avistamiento inicial del áfido, cuando los áfidos de las parcelas donde se aplicaron fungicidas comenzaron a mostrar tasas más altas de infección de la epizootia: micosis de 35.0 y $37.5 \%$ en los tratamientos con ditiocarbamatos y 10.0 y $0.0 \%$ con clorotalonil en 1998 y 1999, respectivamente. Mientras que los áfidos colectados de los testigos sin fungicida mostraron micosis de 87.5 y $85.0 \%$, respectivamente. Al promediar ambas estaciones de cultivo, para la séptima semana el $79.4 \%$ de los áfidos colectados en los tratamientos con fungicidas todavía no mostraban infecciones por Entomophthorales, comparados con 13.8\% de los áfidos colectados de testigos sin fungicida.

2) Se detectó un aumento en la cantidad de áfidos por días observados a lo largo de la estación de cultivo en relación con aquellas parcelas donde no se aplicó fungicida. En 1998, los tratamientos con fungicida promediaron 1,058 áfidos por días más que el testigo sin fungicida y los tratamientos con ditiocarbamatos promediaron 605 áfidos por días más que los tratamientos con clorotalonil. En 1999, los tratamientos con fungicida promediaron 305 áfidos por días más que el testigo sin fungicida y los tratamientos con clorotalonil promediaron 512 áfido por día más que los tratamientos con ditiocarbamatos. Al analizar el área total bajo la curva de progreso de la enfermedad para 1998 y 1999 se determinó que los tratamientos con fungicida (ditiocarbamatos y clorotalonil) promediaron 3.2 veces los áfido por día encontrados en los testigos sin fungicida (Figuras 1C, 1D) (Ruano-Rossil 2001).

Aunque en 1998 el número total de áfidos por día fue más elevado en las parcelas tratadas con fungicidas ditiocarbamatos que con los a base de clorotalonil, en 1999 ocurrió lo contrario y el número de áfidos por día fue más elevado en los tratamientos con clorotalonil. Esta situación puede explicarse con la influencia del clima sobre el fungicida, así como las especies de hongos entomopatógenos que predominaron en cada estación de cultivo y la migración de áfidos a otras plantas. En el desarrollo de una epizootia existen factores ambientales bióticos y abióticos que se consideran importantes. Entre los factores bióticos tenemos las características del hospedero 0 huésped, las características del patógeno, la densidad de la población y la distribución del huésped.

Para las epizootias del AVD, la producción de áfidos alados es un factor ambiental biótico crucial. Entre los factores ambientales abióticos se encuentran la temperatura, luz, fotoperiodo, el suelo, la humedad relativa y la precipitación. En este caso, en 1998 y 1999, uno de los factores ambientales abióticos que más influenció en el desarrollo de las epizootias, así como el número poblacional del AVD y por ende la producción de áfidos alados, fue la precipitación. En 1998, la producción de áfidos alados comenzó temprano debido a una menor precipitación (72.1 mm de lluvia acumulada) en los primeros 30 días después de la llegada del AVD, en comparación con la estación de cultivo de 1999, donde los áfidos migraron tardíamente por la mayor precipitación (152.6 mm de lluvia acumulada) para el mismo periodo.

Para la estación de cultivo de 1998, los tratamientos con clorotalonil tuvieron individuos ápteros (sin alas) con menos micosis y pudieron producir progenie de áfidos alados en altas proporciones y con menos infecciones. Esto les permitió migrar tempranamente fuera de las parcelas experimentales. La producción de áfidos alados y la precipitación no fueron factores significativos en el desarrollo de la epizootia en los tratamientos con clorotalonil, pero sí la humedad relativa y la temperatura. En contraste, en los tratamientos con ditiocarbamatos, los áfidos ápteros tuvieron más micosis y produjeron menos áfidos alados en comparación con clorotalonil. Para los ditiocarbamatos los áfidos alados, la precipitación y la temperatura mostraron ser factores determinantes en su epizootia. Esto tuvo un impacto en su patrón de migración fuera de las parcelas experimentales, donde los áfidos de los tratamientos con ditiocarbamatos tendieron a migrar en una menor proporción que los tratamientos con clorotalonil y a permanecer por más tiempo en la planta de papa. 


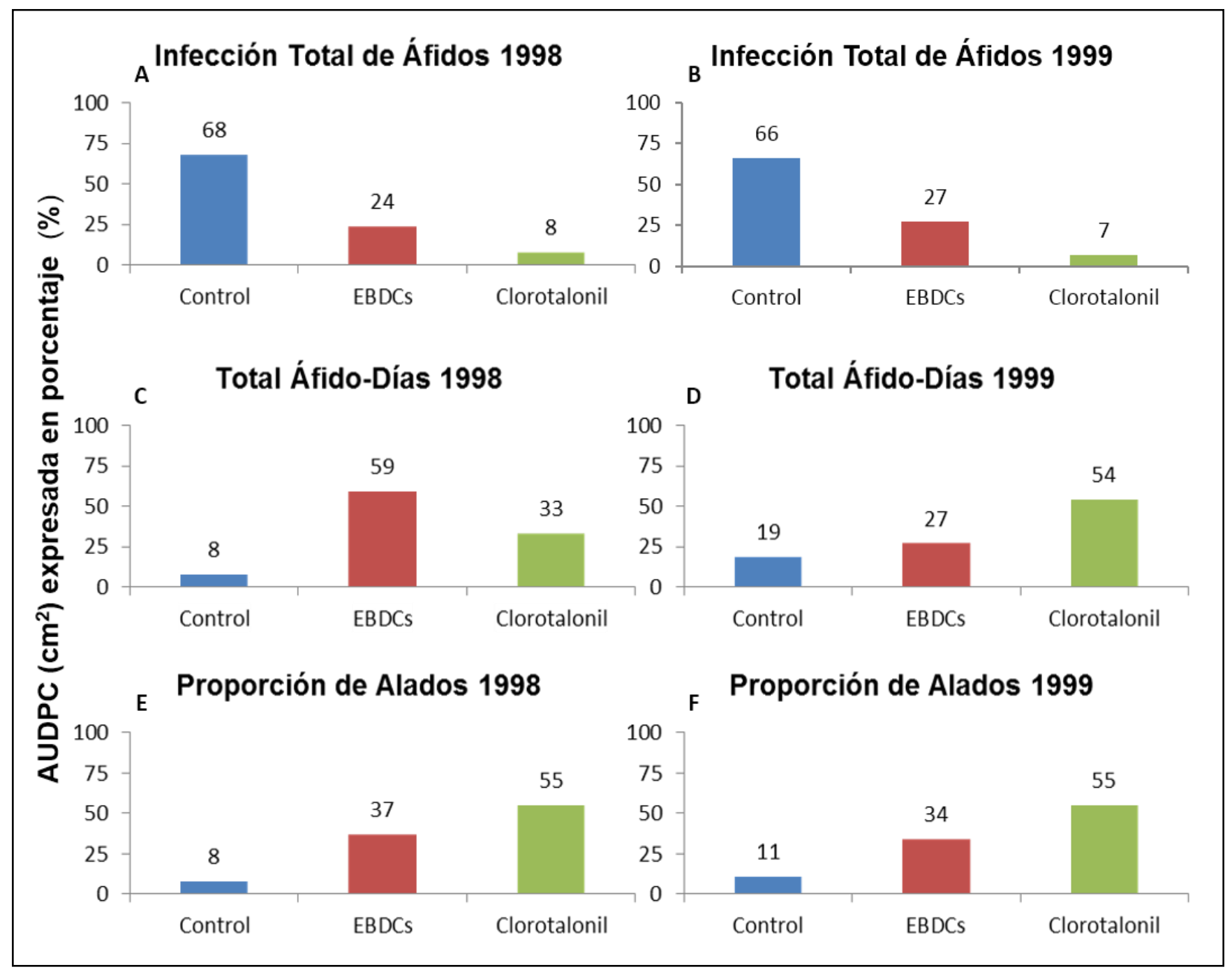

Figura 1. Área total bajo la curva de progreso de la enfermedad (AUDPC) $\left(\mathrm{cm}^{2}\right)$ expresada en porcentaje. A) Infección total de áfidos en 1998. B) Infección total de áfidos en 1999. C) Total de áfido-días en 1998. D) Total de áfido-días en 1999. E) Proporción de áfidos alados en 1998. F) Proporción de áfidos alados en 1999.

En 1999, aunque las condiciones climáticas fueron más húmedas, los tratamientos con clorotalonil prosiguieron ejerciendo un efecto fungicida formidable sobre la micosis de los áfidos ápteros, probablemente por una mejor formulación del fungicida (observaciones de campo Ruano-Rossil). Esto trajo como consecuencia que la población del AVD produjera áfidos alados también con menos infecciones, pero que tendieron a movilizarse fuera de las parcelas más tarde por las condiciones climáticas desfavorables, como la alta precipitación. El análisis estadístico mostró un reverso para esta estación de cultivo, donde la producción de áfidos alados, así como la precipitación, fueron factores significativos en el desarrollo de la epizootia en los tratamientos con clorotalonil. Entre otros factores importantes, tuvimos la humedad relativa, la temperatura y el número poblacional del áfido. En contraste, los tratamientos 
Ruano Rossil. Efectos no Intencionados de Fungicidas para Controlar el Tizón Tardío de la Papa

con ditiocarbamatos para esta estación de cultivo continuaron con niveles más altos de micosis en áfidos ápteros y con menor producción de áfidos alados que los tratamientos con clorotalonil. En este caso, los áfidos alados, la precipitación y la temperatura no fueron factores determinantes en la epizootia de los tratamientos con ditiocarbamatos, pero sí lo fue el número poblacional del áfido.

Para los áfidos provenientes de los testigos sin fungicida, la producción de áfidos alados, la temperatura y la cantidad del áfido fueron los factores significativos para el desarrollo de la epizootia de 1998, mientras que la producción de áfidos alados y la humedad relativa lo fueron para la epizootia de 1999. Ya para la séptima semana después de la llegada del áfido al campo y en los niveles más elevados de micosis del AVD, al promediar ambas estaciones de cultivo, los tratamientos con fungicida tenían $20.6 \%$ de micosis y con promedios poblacionales de 72.0 áfidos por hoja, mientras que los testigos sin fungicida se encontraban en $86.3 \%$ de micosis y 6.2 áfidos por hoja (Ruano-Rossil 2001).

\section{A densidades más elevadas del áfido, mayor producción de áfidos alados}

Los cambios en el contenido nutricional de la planta y la creciente aglomeración de áfidos ápteros promovieron la producción de generaciones de áfidos alados con mayor proporción de áfidos alados en las parcelas donde se aplicaron fungicidas. Al analizar la curva del progreso de la enfermedad para ambas estaciones de cultivo, al usar fungicidas hubo 4.8 veces más la producción de áfidos alados que en los testigos sin fungicida; y clorotalonil produjo 1.5 veces los áfidos alados producidos en los tratamientos con ditiocarbamatos (Figuras 1E, 1F). Ya para la séptima semana después de la llegada del áfido, en los niveles más elevados de micosis del AVD, y al promediar ambas estaciones de cultivo, los ditiocarbamatos, clorotalonil y testigos sin fungicida se encontraban en $36.3,5.0$ y $86.3 \%$ de micosis, respectivamente. Mientras que los niveles de alados producidos en cada grupo se encontraban en 40.0, 70.0 y $10.0 \%$, respectivamente (Ruano-Rossil 2001).

En las parcelas donde no se aplicó fungicida, la producción de áfidos alados y la cantidad de áfidos por día fue mucho menor. Estos individuos alados provenientes de poblaciones de áfidos ápteros infectados y libres de fungicida, también tuvieron mayor tasa de micosis haciéndolos un medio eficiente para la distribución del inóculo a otros áfidos aún no infectados.

En la biología del AVD, la función primordial de los individuos alados es facilitar su movilización fuera de y hacia su hospedero primario, el duraznero o melocotonero (durazno o melocotón). Cuando estos individuos alados vuelan fuera de su hospedero primario, lo hacen hacia hospederos secundarios como la papa. Los hospederos secundarios pueden ser plantas que se encuentran a muy largas distancias, pero aptas para su alimentación y reproducción. Una vez en su hospedero alterno, esta movilización puede ser entre plantas de la misma especie o bien hacia otros hospederos que aseguren su sobrevivencia con una mejor condición nutricional. El insecto, sin embargo, necesitará eventualmente retornar a su hospedero primario para completar su ciclo de vida. M. persicae inverna en forma de huevo en el duraznero, pero durante sus ciclos reproductivos invade muchos hospederos secundarios y se reproduce partenogenéticamente (Raman 1985).

Los áfidos alados también son una fuente importante en la diseminación de sus propios enemigos naturales, como los Entomophthorales, y especialmente si provienen de colonias libres de fungicidas (Shands et al. 1963). En otros sistemas se ha determinado que los áfidos alados son el modo más importante de distribución del inóculo (áfido infectado con hongos entomopatógenos) y cuyo desplazamiento ha sido asociado con grandes epizootias (Rabasse y Robert 1975). Sin embargo, la producción de áfidos alados también representa una fuente importante en la diseminación de fitopatógenos virales de la papa, virus que atacan la planta como el virus del enrollamiento de la hoja (PLRV) y del mosaico Y (PVY). Si a esto aunamos el hecho de que estos áfidos alados pueden provenir de colonias libres de micosis y en proporciones mucho más elevadas, como es el caso de los áfidos provenientes de parcelas donde se aplicaron fungicidas foliares, la transmisión de virus en la papa puede convertirse en un problema aún más severo (Figuras 1E, 1F) (Ruano-Rossil 2001).

\section{Relación entre fungicidas, Entomophthorales y clima sobre la densidad y migración de áfidos}

Las diferencias en el número de áfidos observados al aplicar ditiocarbamatos y a base de 
clorotalonil (Figuras 1C, 1D) (Ruano-Rossil 2001), sugiere que la acción de cada fungicida puede diferir dependiendo de las especies de hongos entomopatógenos presentes en el ambiente y de esas mismas especies de hongo que terminen por convertirse en predominantes durante cada estación de cultivo. Para este caso en particular, los hongos entomopatógenos Pandora neoaphidis (Remaudière y Hennebert) Humber y Entomophthora planchoniana (Cornu) predominaron en 1998, mientras que $P$. neoaphidis fue la única especie que predominó en 1999. Estas mismas diferencias en composición de especies de Entomophthorales coinciden también con diferencias en las condiciones climáticas observadas en cada estación; para ello más cálidas y menos lluviosas en 1998, y más templadas y lluviosas en 1999. No menos importante fue la determinación de una proporción de áfidos alados para ambas estaciones mucho más acentuada en las parcelas donde se aplicó clorotalonil que en aquellas parcelas donde se aplicaron los ditiocarbamatos (Figuras 1E, 1F) (Ruano-Rossil 2001). Sin embargo, esta generación de alados ocurrió más tempro en la estación de 1998 debido a las condiciones climáticas más favorables. La diferencia entre el número de áfidos por día entre los dos grupos de fungicidas (Figuras 1C, 1D), sugiere la existencia de una migración diferencial, donde en 1998 los áfidos de las parcelas con clorotalonil sufrieron menos micosis y lograron migrar más que los áfidos provenientes de las parcelas donde se aplicaron los ditiocarbamatos. En 1999, por condiciones ambientales desfavorables (más bajas temperaturas y más lluvia), la producción de áfidos alados en parcelas con fungicida se produjo tardíamente. Como los áfidos provenientes de parcelas con clorotalonil se encontraban con menos micosis (Figuras 1A, 1B), sus áfido por día también fueron superiores a los áfidos de las parcelas con ditiocarbamatos (Figuras 1C, 1D). A más áfidos por día con menos micosis le prosiguió una mayor proporción de áfidos alados en los tratamientos con clorotalonil (Figuras 1E, 1F).

Las diferencias en la dinámica del crecimiento del AVD arrojan interacciones significativas entre la aplicación de fungicidas foliares, los niveles de micosis en los áfidos, las densidades del áfido alcanzadas, la producción de individuos alados, las condiciones climáticas prevalecientes, así como las especies de hongos entomopatógenos predominantes en cada estación de cultivo. En 1998, cuando los tratamientos con ditiocarbamatos tenían más áfidos, la especie E. planchoniana fue más frecuente al inicio de la estación de cultivo y disminuyó hacia el final de ésta ante la especie $P$. neoaphidis. $P$. neoaphidis fue la especie de entomopatógeno predominante $y$ responsable del 29.9 y el $76.6 \%$ de las micosis en 1998 y 1999 , mientras que E. planchoniana del 13.2 y $8.1 \%$, respectivamente. La precipitación total acumulada en los primeros 30 días después de la llegada del áfido, $72.1 \mathrm{~mm}$ en 1998 y $152.6 \mathrm{~mm}$ en 1999, puede haber influenciado este cambio.

Otros entomopatógenos causantes de micosis fueron Conidiobolus spp., 12.1 y $9.6 \%$, y Zoophthora radicans (Brefeld) 18.4 y $4.6 \%$ de todas las micosis en 1998 y 1999, respectivamente. Especies provenientes de cadáveres que produjeron esporas resistentes no pudieron ser identificadas hasta especie, pero fueron aisladas del 11.1 y $1.1 \%$ de los casos en 1998 y 1999 , respectivamente (Ruano-Rossil 2001, Ruano-Rossil et al. 2004).

\section{Al final un problema complejo}

A través de la investigación de Ruano-Rossil en 2001, se comprobó que aunque los hongos entomopatógenos en las parcelas sin fungicida no permitieron que las poblaciones de áfidos aumentaran exageradamente, los números que alcanzaron estos áfidos aún fueron demasiados elevados como para exceder las tasas de tolerancia permitidas en papa destinada al consumo humano o para semilla, llegando a promedios máximos de 11.7 y 20.6 áfidos por hoja en 1998 y 1999, respectivamente. Por tanto, para esos programas de manejo integrado de plagas que pretendan controlar la diseminación de virus en la papa, su prioridad debe enfocarse en evitar que los áfidos alcancen densidades elevadas. Un aspecto que el estudio de Ruano-Rossil no evaluó fue el efecto combinado de fungicidas con insecticidas sobre los enemigos naturales del AVD. Recientemente se comprobó que dicha interacción fungicida-insecticida, específicamente sobre parasitoides y depredadores del AVD, puede ser un detonante de sus súbitos crecimientos poblacionales (Ragsdale y Koch 2008).

Debido a los problemas de enfermedades foliares de la papa y especialmente por el tizón tardío, así como el control de otros insectos plagas, el producir papa sin tener que aplicar fungicidas e insecticidas se ha convertido en una tarea casi imposible de lograr. Por lo tanto, los programas que tomen en cuenta la conservación y aumento de 
entomopatógenos, la reducción de aplicaciones de fungicidas basados en monitoreo del tizón tardío, principalmente en las primeras semanas del cultivo, así como la incorporación de plaguicidas biológicos, tendrán más probabilidad de éxito en la reducción de la diseminación de los virus de la papa así como la prevención del afianzamiento del AVD como insecto plaga.

Los fungicidas no aumentan directamente las poblaciones del AVD, más que todo su efecto es indirecto al suprimir a sus agentes de control biológico, los Entomophthorales, los cuales pueden mantener sus densidades a bajos niveles. El problema es sin duda complejo pues la aplicación de fungicidas puede alterar diversos sistemas de organismos simultáneamente. Por un lado beneficiando a la planta de papa al controlar fitopatógenos que la atacan, como la Alternaria solani y $P$. infestans, agentes causales de los tizones temprano y tardío, respectivamente. Además, beneficia indirectamente al áfido, aunque por supuesto sin intención, por medio de la supresión de sus hongos entomopatógenos (enemigos naturales) que le infringen tasas de mortalidad significativas. No obstante, en el mismo proceso también existe la posibilidad de favorecer una creciente diseminación de fitopatógenos virales a esas mismas plantas que se tratan de proteger. Esto por promover, obviamente también sin intención, que el insecto permanezca por más tiempo en la planta alimentándose y reproduciéndose con bajas tasas de mortalidad por no sufrir infecciones fungales y por ende aumentando su densidad rápidamente en el campo, así como la promoción de una migración elevada hacia otras plantas.

Esta migración puede ser limitada a plantas cercanas cuando el áfido lo hace caminando, ya sea trasladándose a plantas vecinas cuyas ramas y hojas estén en contacto unas con otras o caminando por el suelo hacia otras plantas más separadas, o bien volando medianas o grandes distancias hacia otras plantas hospederas. De cualquier forma, esta migración, ya sea con o sin alas, es la fuente importante en la propagación de fitopatógenos virales cuando estos insectos adquieren los virus al alimentarse de plantas enfermas y subsecuentemente cuando se convierten en sus mismos vectores al alimentarse de plantas sanas.

\section{Literatura Citada}

American Public Health Association. 2004. Edición original en Inglés: Control de Communicable Diseases Manual, 18th Edition. Infecciones por Entomophthorales: Conidiobolomicosis, p. 61.

Dedryver, C.A. 1980. Preliminary results concerning the role of three species of Entomophthora in limiting populations of cereal aphids in western France. Bull. SROP 3: 5-9.

Fedorenko, V.P. 1985. Factors limiting the injuriousness of the beet aphid. Zashchita Rastenii 12: 12-13.

Feng, M.G., C.L. Liu, J.H. Xu, Q. Xu. 1998. Modeling and biological implication of time-dose-mortality data for the entomophthoralean fungus, Zoophthora anhuiensis, on the green peach aphid Myzus persicae. Journal of Invertebrate Pathology 72: 246-251.

Feng, M.G., J.B. Johnson, S.E. Halbert. 1991. Natural control of cereal aphids (Homoptera: Aphididae) by entomopathogenic fungi (Zygomycetes: Entomophthorales and parasitoids (Hymenoptera: Braconidae and Encyrtidae) on irrigated spring wheat in southwestern Idaho. Environmental Entomology 20: 1699-1710.

Forgash, A.G. 1985. Insecticide resistance in the Colorado potato beetle. In Ferro, D.N. and R.H. Voss (eds.) Proceedings of the Symposium on the Colorado potato beetle, XVII International Congress of Entomology.

Fuxa, J.R., Y. Tanada. 1987. Epidemiological concepts applied to insect epizootiology, pp. 3-21. In J.R. Fuxa and Y. Tanada [eds.], Epizootiology of insect diseases, New York: Wiley.

Goodwin S.B., L.S. Sujkowski, W.E. Fry. 1996. Widespread distribution y probable origin de resistance to metalaxyl en clonal genotypes de Phytophthora infestans in the United States y western Canada. Phytopathology 86: 793-800.

Hofmaster, R.N., R.L. Waterfield, J.C. Boyd. 1967. Insecticides applied to the soil for control of eight species of insects on Irish potatos in Virginia. Journal of Economic Entomology 60: 1311-1318.

Horn, D.J. 1983. Selective mortality of parasitoids and predators of Myzus persicae on collards treated with malathion, carbaryl or Bacillus thuringiensis. Entomologia Experimentalis et Applicata 34:208-211.

Klingen, I., Haukeland S. 2006. The soil as a reservoir for natural enemies of pest insects and mites with emphasis on fungi and nematodes. In: Eilenberg J, Hokkanen HMT (eds) An ecological and societal approach to biological control. Springer, Berlin, 145-212.

Lagnaoui, A., E.B. Radcliffe. 1998. Potato fungicides interfere with entomopathogenic fungi impacting population dynamics of green peach aphid. American Journal of Potato Research 75: 19-25. 
Milner, R.J., R.E. Teakle, G.G. Lutton, F.M. Dare. 1980. Pathogens (Phycomycetes: Entomophthoraceae) of the blue-green aphid Acyrthosiphon kondoi Shinji and other aphids in Australia. Australian Journal of Botany 28: 601-619.

Nielsen, C., A. E. Hajek. 2005. Control of Invasive Soybean Aphid, Aphis glycines (Hemiptera: Aphididae), Populations by Existing Natural Enemies in New York State, with Emphasis on Entomopathogenic Fungi. Environmental Entomolology 34 (5): 1036-1047.

Pell, J.K., J.J. Hannam, D.C. Steinkraus. 2010. Conservation biological control using fungal entomopathogens. Biocontrol 55: 187-198.

Quinton, R.J. 1955. DDT-resistant Colorado potato beetles? Proceeding of the North Central Entomological Society of America 9: 94-95.

Rabasse, J.M., Y. Robert. 1975. Factors limiting populations of Aphis fabae in western France. IIIncidence of infection by Entomophthora on primary hosts and spring beans. Entomophaga 20: 49-63.

Radcliffe, Ted, Dave Ragsdale, Abdelaziz Lagnaoui. 1996. Los Fungicidas Afectan el Control de Áfidos. Reproducido con permiso de Valley Potato Grower@ 1996 Red River Valley Potato Growers Association. In: Radcliffe's El Texto Mundial de MIP. http://ipmworld.umn.edu/cancelado/Spchapters/Lagnao uiSp.htm

Ragsdale, D.W., K.A. Koch. 2008. Fungicides: Do They Adversely Affect Beneficial Insect Pathogens in Multiple Cropping Systems? Proceedings of the 2008 Illinois Crop Protection Technology Conference, p. 57-66. http://www.ipm.uiuc.edu/education/proceedings/icptcp20 08.pdf
Raman, K.V. 1985. Transmisión de Virus de Papa por Áfidos. Boletín de Información Técnica 2. Centro Internacional de la Papa (CIP). p. 23.

Ruano-Rossil, J.M. 2001. Suppression of Entomopathogenic Fungi of Green Peach Aphid, Myzus persicae (Sulzer), by Late Blight Fungicides. Ph.D. thesis research dissertation, University of Minnesota). $98 \mathrm{p}$.

Ruano-Rossil, J.M., E.B. Radcliffe, D.W. Ragsdale. 2004. Disruption de entomopathogenic fungi de green peach aphid, Myzus persicae (Sulzer), by fungicides used to control potato late blight. In: Simon J.C., Dedryver C.A., Rispe C. and Hullé M. (eds), Aphids in a New Millennium, INRA, Versailles, p. 365-370.

Shands, W.A., G.W. Simpson, I.M. Hall. 1963. Importance de entomogenous fungi in controlling aphids on potatoes in northeastern Maine. Maine Agriculrural Experimental Station Technical Bulletin 6, 42 p.

Soper, R.S., D.M. MacLeod. 1981. Descriptive epizootiology of an aphid mycosis Entomophthora canadensis. Technical Bulletin USDA 1632: 17.

Tanada, Y., H.K. Kaya. 1993. Insect pathology. San Diego Academic Press. 666 p.

Wraight, S.P., T.J. Poprawski, W.L. Meyer, F.B. Peairs. 1993. Natural enemies of Russian wheat aphid (Homoptera: Aphididae) and associated cereal aphid species in spring-planted wheat and barley in Colorado. Environmental Entomology 22: 1383-1391.

Sometido para publicación el 10 de marzo de 2015. Aceptado para publicación el 16 de julio de 2015. 